\title{
Editorial
}

\section{Acute Kidney Injury and Renal Replacement Therapy: ADOPPS Wanted!}

\author{
Claudio Ronco \\ Department of Nephrology, Dialysis and Transplantation, International Renal Research Institute of Vicenza (IRRIV), \\ San Bortolo Hospital, Vicenza, Italy
}

With this issue, Blood Purification will begin a series of editorials from the Editor in Chief dedicated to hot topics and areas of research where advances and new ideas are needed. This coincides with the birth of 'Cappuccino with Claudio Ronco', a club of Blood Purification that in a short YouTube clip will discuss a paper published in this journal and hopefully stimulate interest and new ideas especially in the younger generation of readers and investigators.

Acute kidney injury (AKI) is becoming a major dilemma for healthcare systems not only because of the high cost in terms of healthcare resources required to deal with it, but also because evidence is growing that AKI may have a significant impact on long-term outcomes. With regard to $\mathrm{CKD}, \mathrm{AKI}$ is associated not only with the development of de novo disease, but also with the worsening of preexisting disease - both these phenomena may lead to ESRD. ESRD (i.e., dialysis dependence) and progression and worsening of CKD represent two of the most important aspects to consider. Furthermore, the presence of AKI and the requirement of renal replacement therapy (both short- and long-term) represent clear risk factors for mortality and increased healthcare expenditure.

Under these circumstances, it seems logical to issue a 'call to action' to align all scientific, academic, and clinical efforts designed to improve the outcomes of AKI patients. An important aspect here is to partner with healthcare providers, governments, and important stakeholders in the lay community to ensure a broad-based response to this disorder. Unfortunately, the medical community has only recently stressed the importance of AKI as a syndrome, and is still making distinctions and objections to consensus, classifications and definitions provided by experts. In particular, some disciplines appear to be skeptical about the use of widely accepted and clinically validated terminology, leaving the real epidemiology of the syndrome highly biased and uncertain. As a concrete example, in some institutions or geographical areas, considerable disagreement remains on relatively basic issues such as the use of the term 'acute kidney injury' and characterization of the syndrome depending on whether or not the patient requires renal replacement therapy. This lack of therapeutic consensus has caused confusion in the clinical community about the true incidence of AKI and its associated outcomes.

All of these concepts are further complicated by the lack of strong evidence supporting the different therapeutic strategies. While this is true for almost every treatment provided in the ICU, this lack of definitive evidence has a particularly striking effect on renal support/substitution in critically ill patients with AKI. Although consensus statements are provided by current national or international guidelines, few of them can be easily incorporated into a bundle or treatment algorithm due to the fact that they are highly debated by the clinical community and are mostly the result of expert opinion.

\section{KARGER}

E-Mail karger@karger.com

www.karger.com/bpu (c) 2014 S. Karger AG, Basel

0253-5068/14/0374-000I\$39.50/0
Claudio Ronco, MD

Department of Nephrology, Dialysis and Transplantation International Renal Research Institute of Vicenza (IRRIV)

Viale Rodolfi 37, IT-36100 Vicenza (Italy)

E-Mail cronco@goldnet.it 
What should we do then? Should this lack of agreement, consensus, and evidence be the basis for a nihilistic approach encompassing therapy denial to many patients or groups of patients? Should we stop research and technological advancements because there is little chance that they will ultimately influence clinical practice or outcomes? Should we withdraw from the field assuming that a particular treatment makes little or no difference? Well, we should learn from our past experiences and try to use these lessons for shaping our future.

There was a moment in chronic dialysis when, in spite of technological advances and improvements in biomaterials, the outcomes of patients in terms of morbidity and mortality were highly variable from center to center and region to region. These results led to the impression that unmeasured variables such as practice patterns could have an impact on the final outcome.

Based on this concept, DOPPS (Dialysis Outcome Practice Pattern Study) was created to ascertain whether or not practice patterns could influence the differences in observed clinical results [1]. DOPPS was conceived as a prospective cohort study of hemodialysis practices based on the collection of observational longitudinal data for a representative random sample of patients from dialysis facilities in more than 20 countries. The samples of patients in each facility, and sample of facilities in each country, were designed to provide a reliable picture of practices and outcomes at the respective levels. The study was designed to help researchers describe differences in practice patterns that could potentially correlate with differences in outcomes and to identify treatment-related factors that potentially could be modified to improve patient care and outcomes.

The study was so successful that a subsequent international version (iDOPPS) was created and even a specific peritoneal dialysis study was performed (PDOPPS) [2]. The scope of iDOPPS was to provide information about the high variability in practice patterns and outcomes that can be observed in any single country and also in different countries. This increased variation along with the study's large sample size has led to a greatly enhanced ability to understand the relationships between various treatment effects and patient outcomes.

Today, the DOPPS annual report includes a comprehensive collection of descriptive statistics spanning more than a decade of longitudinal DOPPS data collection. Calculated for multiple, representative cross-sectional cohorts, each annual report portrays country-specific results and illustrates trends over several practice areas.
With this in mind and based on the success of the DOPPS initiative, it seems almost inevitable to consider the possibility to develop an ADOPPS (Acute Dialysis Outcome Practice Pattern Study) where all factors affecting acute patient outcome can be analyzed and scored in terms of importance. While this has been the principle of the ADQI initiative [3] since its inception, a more specific project focusing on practice patterns as possible variables affecting outcome in critically ill patients is needed. Hopefully, the scientific community will use this provocative editorial to initiate a pathway toward the development of such an initiative. ADOPPS may help answer several questions that are still unanswered today. For example, how important is the cooperation of nephrologists and intensivists in the management of renal replacement therapy in the acute patient? [4]. How much weight on outcome can a cooperative approach like the one described in the Vicenza Model have? [5]. Do the procedures for vascular access creation affect outcome? Is anticoagulation modality important? Does the decision-making process that is typical of a single unit make any difference in the final results? What is the real dose prescription and delivery in practice? This call to action represents an invitation to a wide spectrum of specialists to acknowledge that AKI is a complex field for which a consistent and systematic clinical approach is still desperately needed. The achievement of such an approach will not be easy due to the multifactorial nature of the syndrome and the disparate clinical care environments in which AKI patients receive treatment around the world. Nevertheless, if we collaborate to optimize our clinical practices, our patients will be the ones who stand to benefit.

References

\footnotetext{
Pisoni RL, Gillespie BW, Dickinson DM, Chen K, Kutner MH, Wolfe RA: The Dialysis Outcomes and Practice Patterns Study (DOPPS): design, data elements, and methodology. Am J Kidney Dis 2004;44(suppl 2):7-15.

-2 Perl J, Robinson B, Davies S; International Society for Peritoneal Dialysis, Arbor Research Collaborative, and the PDOPPS investigators: Update on the Peritoneal Dialysis Outcomes and Practice Patterns Study (PDOPPS). Perit Dial Int 2014;34:332.

-3 Ronco C, Kellum JA, Mehta R: Acute Dialysis Quality Initiative (ADQI). Nephrol Dial Transplant 2001;16:1555-1558.

-4 Flores-Gama C, Merino M, Baranda F, Cruz DN, Ronco C, Vazquez-Rangel A: The impact of integrating nephrologists into the postoperative cardiac intensive care unit: a cohort study. Cardiorenal Med 2013;3:79-88.

$\checkmark 5$ Ronco C: Critical care nephrology: can we clone the 'Vicenza Model'? Int J Artif Organs 2007;30:181-182.
} 